
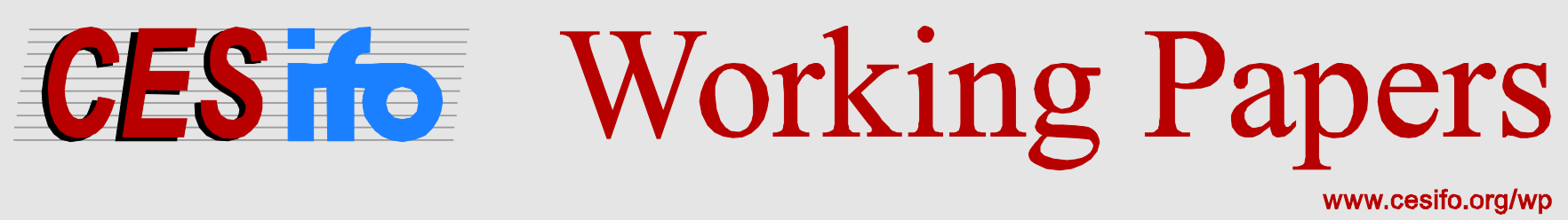

\title{
Are Sequential Round-Robin Tournaments Discriminatory?
}

\author{
Marco Sahm
}

CESIFO WORKING PAPER NO. 6421

CATEgory 2: PUBliC CHOICE

MARCH 2017

An electronic version of the paper may be downloaded

- from the SSRN website:

- from the RePEc website:

- from the CESifo website:

www.SSRN.com

www.RePEc.org

www.CESifo-group.org/wp 


\title{
Are Sequential Round-Robin Tournaments Discriminatory?
}

\begin{abstract}
I examine sequential round-robin tournaments with three and four symmetric players. Each player is matched once with each other player. If the matches are organized as Tullock contests (all-pay auctions), the tournament will be almost fair (highly discriminatory): subject to the position of their matches in the sequence of the tournament, the differences in players' ex-ante winning probabilities and expected payoffs will be small (large). The differing results originate from the higher discriminating power of the all-pay auction. Moreover, the resulting discouragement effect in tournaments with all-pay auctions implies lower aggregate effort than in tournaments with suitable Tullock contests. The fairness of round-robin tournaments may be improved by the use of an endogenous sequence of matches or the requirement that players fix their effort ex-ante.
\end{abstract}

JEL-Codes: C720, D720, Z200.

Keywords: sequential round-robin tournament, contest success function, discriminatory power, Tullock contest, all-pay auction.

\author{
Marco Sahm \\ University of Bamberg \\ Department of Economics \\ Feldkirchenstraße 21 \\ Germany - 96047 Bamberg \\ Marco.Sahm@uni-bamberg.de
}

I would like to thank Nina Flügel, Hermann Freibott, Simon Heimgärtner, Christoph Laica, and Arne Lauber for excellent research assistance. 


\section{Introduction}

A round-robin tournament, also referred to as all-play-all tournament, is a form of competition in which each participant is matched with all other participants in turn. Important scientific applications of round-robin tournaments include the study of voting theory and social choice theory, e.g. for testing the existence of a Condorcet winner. In practice, round-robin tournaments are widely used as an organizational form of competition, particularly in sports. Most sports leagues around the world are - at least partially organized as some version of a round-robin tournament. Prominent examples of double round-robin tournaments include the major European football leagues in England, Spain, Germany, and Italy. Moreover, smaller pure round-robin tournaments are embedded in early stages of many multi-stage sports tournaments, e.g. the first round (group stage) of the FIFA World Cup (since 1950) and UEFA European Championship (since 1980) with four teams per group, or the second round of the FIFA World Cup (1982) with three teams per group. ${ }^{1}$ In tournaments with three teams, the natural schedule of the matches is sequential. And even in tournaments with four teams, the matches are mostly scheduled sequentially due to technological constraints (better accessibility) or economic considerations (higher profitability).

One reason for its high popularity may be the common wisdom according to which "a round-robin tournament is the fairest way to determine the champion among a known and fixed number of participants. Each participant, player or team, has equal chances against all other opposites" (Wikipedia ${ }^{2}$, 2016). This paper addresses the question whether this common wisdom is true. Are round-robin tournaments really fair? Or is their sequential structure inherently discriminatory?

Despite the practical and scientific relevance of round-robin tournaments, the economic analysis of sequential contests has mainly focussed on other forms of dynamic battles like elimination tournaments or races. ${ }^{3}$ An exemption is the article by Krumer et al. (2017) who investigate the winning probabilities and expected payoffs in sequential round-robin tournaments with three and four symmetric players. The different pairwise matches take place one after the other and the player with the most victories wins the tournament. The authors assume that each single match is organized as an all-payauction and find substantial discrimination by the order of matches in the

\footnotetext{
${ }^{1}$ In January 2017 FIFA decided that, from 2026 on, it will extend its World Cup to 48 teams and organize the first round in form of round-robin tournaments with three teams per group.

${ }^{2}$ Accessed at https://en.wikipedia.org/wiki/Round-robin_tournament on 16/12/2016

${ }^{3}$ The related literature is summarized, for example, by Konrad (2009, Chapter 8).
} 
subgame perfect equilibrium of the sequential game. For example, they show that in the 3-player tournament, the player who competes in the first and last match has a much higher winning probability and expected payoff than the two other players.

These findings are in stark contrast to the common wisdom of fairness. The intuition is that the sequential structure implies an asymmetry in intermediate scores which discourages trailing participants from providing equivalent effort. ${ }^{4}$ In the model of Krumer et al. (2017), this discouragement effect is particularly strong because the contest success function of an all-pay auction that determines the players' winning probabilities in each single match of the tournament is fully discriminating in the sense that the player with the highest effort wins for sure. This raises the question whether the sequential round-robin tournament will be fairer if its matches are organized as contests with less discriminating contest success functions.

In this paper, I address the fairness issue under the assumption that the matches of the sequential round-robin tournament are organized as Tullock contests with discriminating powers (i.e. exponents) which are sufficiently low to guarantee the existence of a unique subgame perfect equilibrium in pure strategies. I find that such Tullock tournaments are discriminatory as well. Quantitatively, however, the extent of discrimination by the order of matches is much smaller with Tullock contests than with all-pay auctions. The sequential round-robin tournament with matches organised as Tullock contests is almost fair in the sense that both, the expected payoffs and the winning probabilities do not differ much between players. Intuitively, the discouragement of trailing participants will be mitigated if the respective contests are less discriminating because the winning probability and expected payoff of a player is positive even if he invests less than his opponent.

The asymmetries between the players' expected payoffs and winning probabilities in the subgame perfect equilibrium of round-robin tournaments with Tullock contests differ from those with all-pay auctions not only quantitatively but also qualitatively. For example, in the 3-player tournament with Tullock contests of moderate discriminating power, the player who competes in the first and last match has the lowest winning probability and expected payoff - the diametric result to what Krumer et al. (2017) find for tournaments with all-pay auctions. The analysis shows that the discriminating power of the contest success function impacts not only the extent but also the direction of discrimination within the tournament.

A significant part of the literature on contests deals with the issue of

\footnotetext{
${ }^{4}$ Similar discouragement effects occur in most forms of dynamic competition (Konrad, 2009, Chapter 8).
} 
rent dissipation. In a static winner-take-all contest between a finite number of symmetric players, the rent is fully dissipated in an all-pay auction but only partially in a Tullock contest. I show that, by contrast, expected aggregate effort in sequential round-robin tournaments with all-pay auctions may be lower than in sequential round-robin tournaments with Tullock contests due to stronger discouragement effects in the former. This implies that the contest designer can choose sequential round-robin tournaments with Tullock contests which are not only closer but also more intense than sequential round-robin tournaments with all-pay auctions.

The above results of discrimination rely on the assumption that the tournament follows an exogenously fixed sequence of matches. Considering the 3-player tournament, I illustrate that the fairness of the tournament can be easily improved by endogenizing the sequence of matches such that the winner of the first match must play also in the second match - regardless of whether the matches are organized as Tullock contests or all-pay auctions.

In some round-robin tournaments, a tight time schedule does not allow players to adapt their effort level from match to match. Instead, they must fix their investment before the tournament starts and then compete all of their matches at the same level. Considering the 3-player tournament, I show that the requirement of such ex-ante effort provision eliminates any discrimination.

The remainder of this paper is organized as follows. Section 2 describes the general model of the round-robin tournament and its matches. In Section 3 and 4, I analyze round-robin tournaments with three and four players, respectively. Section 5 concludes.

\section{The Tournament and the Matches}

I consider sequential round-robin tournaments with $n \in\{3,4\}$ symmetric, risk-neutral players. Successively, each player is matched one-to-one with each other player. ${ }^{5}$ The player with the most victories out of the $\frac{n(n-1)}{2}$ different pairwise matches wins the tournament. If there are $m \in\{1, \ldots, n\}$ players sharing the maximum number of victories, each of them wins the tournament with probability $1 / m$. The value of winning the tournament is

\footnotetext{
${ }^{5}$ Notice that, in a round-robin tournament with $n \geq 3$ players in which the $\frac{n(n-1)}{2}$ different pairwise matches are scheduled sequentially, there are $S(n)=\frac{[1 / 2 \cdot n(n-1)] !}{n !}$ different sequences except for renaming players. With $S(3)=1, S(4)=30$, and $S(5)=30240$, the rapid increase of $S(n)$ in $n$ highlights that a comprehensive analysis of round-robin tournaments with more than four players requires enormous processing power.
} 
identical for all players and normalized to $1 .^{6}$

Each match of the tournament is organized as a Tullock contest between two players, $A$ and $B$, with linear costs of effort, see e.g. Konrad (2009, Chapter 2.3). More specifically, player $A$ 's probability of winning match $k$ is

$$
p_{A}^{k}=\left\{\begin{array}{cc}
1 / 2 & \text { if } x_{A}^{k}=x_{B}^{k}=0, \\
\frac{\left(x_{A}^{k}\right)^{r}}{\left(x_{A}^{k}\right)^{r}+\left(x_{B}^{k}\right)^{r}} & \text { else, }
\end{array}\right.
$$

where $x_{i}^{k}$ denotes the effort of player $i \in\{A, B\}$ in match $k$, and $r \geq 0$ describes the discriminating power of the contest. In the context of rent-seeking, this type of contest success function was introduced by Tullock (1980) and given an axiomatic foundation by Skaperdas (1996). For $r=0$ the players' winning probabilities are independent from efforts and equal $1 / 2$. For $r=1$ the contest is also referred to as lottery contest. For $r \rightarrow \infty$ the winning probability of the player with the higher investment approaches 1, i.e. the contest success function converges to the perfectly discriminating all-pay auction.

Player $A$ chooses $x_{A}^{k}$ in order to maximize his expected payoff

$$
E_{A}^{k}=p_{A}^{k}\left(w_{A}^{k}-x_{A}^{k}\right)+\left(1-p_{A}^{k}\right)\left(\ell_{A}^{k}-x_{A}^{k}\right),
$$

where $w_{i}^{k}$ denotes player $i$ 's expected continuation payoff from winning match $k$ and $\ell_{i}^{k}$ denotes his expected continuation payoff from losing match $k$, with $w_{i}^{k} \geq \ell_{i}^{k} \geq 0$ for $i \in\{A, B\}$. For $w_{A}^{k}=\ell_{A}^{k}$, the optimal choice is $x_{A}^{k}=0$ for any $x_{B}^{k} \geq 0$. If $x_{A}^{k}=0$ and $w_{B}^{k}>\ell_{B}^{k}$, player $B$ will have no best reply unless there is a smallest monetary unit $\varepsilon>0$; the best reply is then $x_{B}^{k}=\varepsilon$. As $\varepsilon \rightarrow 0$, in the limit, $x_{B}^{k} \rightarrow 0$ and $p_{B}^{k} \rightarrow 1$. Otherwise, a unique Nash equilibrium in pure strategies exists if and only if the discriminating power of the contest is sufficiently small (Nti, 1999); more precisely if and only if for $i, j \in\{A, B\}$ with $i \neq j$ and $w_{i}^{k}-\ell_{i}^{k}=\min \left\{w_{A}^{k}-\ell_{A}^{k}, w_{B}^{k}-\ell_{B}^{k}\right\}$ the inequality

$$
r \leq 1+\left(\frac{w_{i}^{k}-\ell_{i}^{k}}{w_{j}^{k}-\ell_{j}^{k}}\right)^{r}
$$

is satisfied. I will restrict the analysis below to these cases and compare them to the case in which $r \rightarrow \infty$, i.e. the all-pay auction as analyzed by Krumer et al. (2017). The equilibrium effort levels can then be derived from the necessary conditions

$$
\frac{\partial E_{i}^{k}}{\partial x_{i}^{k}}=\frac{r\left(x_{i}^{k}\right)^{r-1}\left(x_{j}^{k}\right)^{r}}{\left[\left(x_{i}^{k}\right)^{r}+\left(x_{j}^{k}\right)^{r}\right]^{2}}\left(w_{i}^{k}-\ell_{i}^{k}\right)-1=0
$$

\footnotetext{
${ }^{6}$ For risk-neutral players with identical valuations, the tie breaking rule is equivalent to the assumption that the prize money is shared equally among the players with the maximum number of victories.
} 
yielding

$$
x_{i}^{k}=r \frac{\left(w_{i}^{k}-\ell_{i}^{k}\right)^{1+r}\left(w_{j}^{k}-\ell_{j}^{k}\right)^{r}}{\left[\left(w_{i}^{k}-\ell_{i}^{k}\right)^{r}+\left(w_{j}^{k}-\ell_{j}^{k}\right)^{r}\right]^{2}}
$$

for $i, j \in\{A, B\}$ with $i \neq j$. The resulting equilibrium winning probabilities equal

$$
p_{i}^{k}=\frac{\left(w_{i}^{k}-\ell_{i}^{k}\right)^{r}}{\left(w_{i}^{k}-\ell_{i}^{k}\right)^{r}+\left(w_{j}^{k}-\ell_{j}^{k}\right)^{r}} .
$$

Inserting (3) and (4) into (1) yields the expected equilibrium payoffs.

\section{The Tournament with Three Players}

In this section, I consider round-robin tournaments with three players. I first determine the subgame perfect equilibrium of the tournament with an exogenously given sequence of matches organized as Tullock contests, henceforth referred to as Tullock tournaments. I then use the results to discuss the issues of fairness and effort provision, particularly in comparison to tournaments with matches organized as all-pay auctions, henceforth referred to as APA-tournaments.

\subsection{Tullock tournaments: analysis and equilibria}

In the tournament with three players, there is a unique sequence of matches (except for renaming players). Hence, without loss of generality, I consider the sequence in which first player 1 is matched with player 2 , second player 1 is matched with player 3 , and third player 2 is matched with player 3 . The structure of the resulting sequential game is depicted in Figure 1. The six nodes $k \in\{A, \ldots, F\}$ represent the non-trivial combinations for which the winner of the tournament has not yet been determined when the respective match starts.

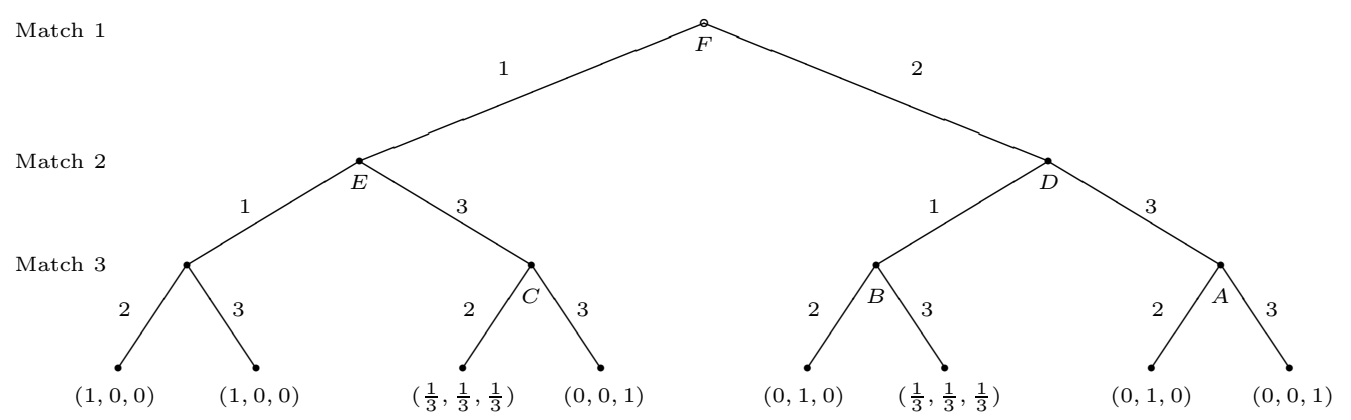

Figure 13 -player round-robin tournament with exogenous sequence 
The game is solved by backward induction for its subgame perfect equilibrium, making repeatedly use of equations (3), (4), and (1). Appendix A illustrates this procedure step by step for $r=1$, i.e. for the tournament with matches organized as lottery contests, henceforth referred to as lottery tournament $(L C)$. It turns out that condition (2) for the existence of a Nash equilibrium in pure strategies will be satisfied in each match of the tournament ${ }^{7}$ (and thus the tournament will have a unique subgame perfect equilibrium in pure strategies) if and only if the discriminating power does not exceed the threshold $\bar{r} \approx 1.383$, implicitly defined by $\bar{r}=1+1 / 2^{\bar{r}}$. Therefore, I restrict the following analysis to $r \leq \bar{r}$.

Figure 2 (3) displays the equilibrium values of the players' ex-ante winning probabilities (expected payoffs) as functions of the discriminating power $r .{ }^{8}$ Four observations are striking. First, in contrast to common wisdom, whenever the contest success function has positive discriminating power, the tournament is discriminatory: there is no $r>0$ such that the ex-ante winning probabilities or expected payoffs of all three players coincide. Second, the level of discrimination increases with the discriminating power of the contest success function: the relative standard deviation of ex-ante winning probabilities (expected payoffs) increases monotonically from $0 \%$ for $r=0$ to $10.53 \%(36.66 \%)$ for $r=1.38$. Third, these figures show that, whenever the discriminating power of the contest success function is sufficiently low to guarantee a unique subgame perfect equilibrium in pure strategies, the extent of discrimination is small. Finally, not only the level but also the direction of discrimination varies with the discriminating power of the contest success function: If $r$ is below (above) a certain threshold, player 3 (1) has the highest ex-ante winning probability and expected payoff. I summarize these results in

Proposition 1 Suppose that $r \leq \bar{r}$. Then the sequential round-robin tournament with three symmetric players and matches organized as Tullock contests has a unique subgame perfect equilibrium with the following properties:

(a) If $r>0$, the tournament is discriminatory.

(b) The level of discrimination as measured by the relative standard deviation of ex-ante winning probabilities (expected payoffs) increases in $r$.

\footnotetext{
${ }^{7}$ In fact, the smallest ratio $\frac{w_{i}^{k}-\ell_{i}^{k}}{w_{j}^{k}-\ell_{j}^{k}}$ arises in nodes $B$ and $C$ with $\frac{1 / 3-0}{1-1 / 3}=\frac{1}{2}$.

${ }^{8}$ The graphs of Figures 2 and 3 interpolate the results from a simulation which stepwise computes the equilibria increasing $r$ from 0 to 1.38 in steps of 0.01 .
} 


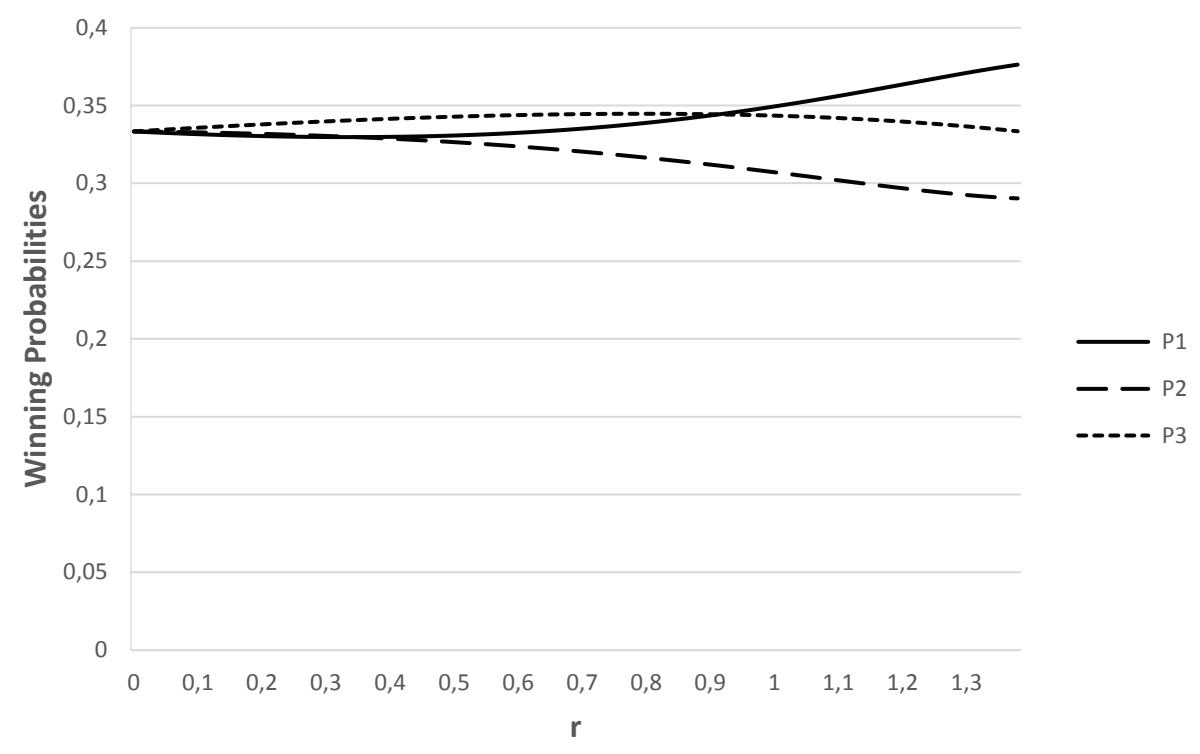

Figure 2 Ex-ante winning probabilities as functions of $r$

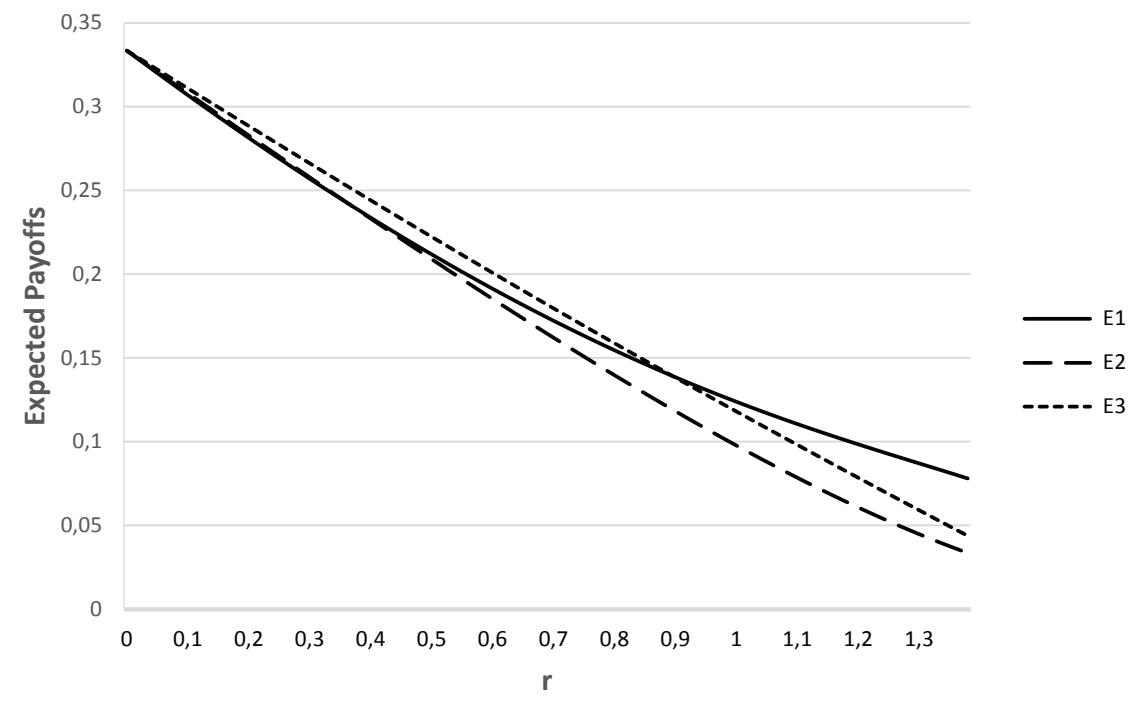

Figure 3 Expected payoffs as functions of $r$ 
(c) The extent of discrimination is small: the relative standard deviation of ex-ante winning probabilities (expected payoffs) is less than $10.53 \%$ (36.66\%).

(d) If $r$ is below (above) a certain threshold $\tilde{r}$, player 3 (1) has the highest ex-ante winning probability and expected payoff.

\section{$3.2 \quad$ Fairness}

From the viewpoint of contest design, the fairness of competition, also referred to as competitive balance, is not only a matter of justice but also a marketing instrument that helps to create thrill and attract attention. For example, attracting more viewers and thereby sponsors, a closer sports tournament may also yield higher revenues and profits (Szymanski and Késenne, 2004). In this subsection, I discuss the fairness issue in more detail.

\subsubsection{Tullock tornaments vs. APA-tournaments}

In comparison to the findings by Krumer et al. (2017) for matches organized as all-pay auctions, the results for matches organized as lottery contests differ fundamentally in both, the quality and the quantity of discrimination induced by the sequential order of matches. The columns labelled exogenous of Tables 1 and 2 summarize the equilibrium values of the players' ex-ante winning probabilities and expected payoffs for the APA-tournament (Krumer et al., 2017) and the Lottery tournament (LC), i.e. the Tullock tournament with $r=1$.

Table 1 Winning probabilities in 3-player tournaments

\begin{tabular}{l|cc|cc}
\hline \hline sequence & \multicolumn{2}{|c|}{ exogenous } & \multicolumn{2}{c}{ endogenous WF } \\
\hline matches & APA & LC & APA & LC \\
\hline player 1 & 0.193 & 0.352 & 0.348 & 0.339 \\
player 2 & 0.682 & 0.305 & 0.348 & 0.339 \\
player 3 & 0.125 & 0.343 & 0.303 & 0.322 \\
\hline rel. stand. dev. & 0.7443 & 0.0612 & 0.0643 & 0.0230 \\
\hline \hline
\end{tabular}

Quantitatively, the APA-tournament is much more discriminatory than any Tullock tournament with $r \leq \bar{r}$ : While the relative standard deviation of ex-ante winning probabilities (expected payoffs) varies between $0 \%$ and $10.53 \%(36.66 \%)$ for the Tullock tournaments, the relative standard deviation 
Table 2 Expected payoffs in 3-player tournaments

\begin{tabular}{l|cc|cc}
\hline \hline sequence & \multicolumn{2}{|c|}{ exogenous } & \multicolumn{2}{c}{ endogenous WF } \\
\hline matches & APA & LC & APA & LC \\
\hline player 1 & 0.0833 & 0.1256 & 0 & 0.1154 \\
player 2 & 0.4167 & 0.0979 & 0 & 0.1154 \\
player 3 & 0 & 0.1180 & 0 & 0.1082 \\
\hline rel. stand. dev. & 1.0801 & 0.1027 & 0 & 0.0300 \\
$\sum$ expected payoffs & 0.5 & 0.3415 & 0 & 0.3390 \\
\hline \hline
\end{tabular}

of ex-ante winning probabilities (expected payoffs) equals $74.43 \%(108.01 \%)$ for the APA-tournament.

Proposition 2 Suppose that there are 3 symmetric players and $r \leq \bar{r}$. Then the Tullock tournament is less discriminatory than the APA-tournament as measured by both, the relative standard deviation of ex-ante winning probabilities and the relative standard deviation of ex-ante expected payoffs.

Qualitatively, in the APA-tournament, the player who competes in the first and last match (player 2) has the highest winning probability and expected payoff by far, whereas the player who competes in the two last matches (player 3) has the lowest winning probability and expected payoff. By contrast, as Figures 2 and 3 show, player 2 will be the least favored player for Tullock contests with sufficiently large $r$, and player 3 will be the most favored player for Tullock contests with sufficiently small $r$. The intuition is that the sequential structure implies an asymmetry in intermediate scores that discourages trailing players from providing equivalent effort. In the APA-tournament, this discouragement effect is particularly strong due to the perfectly discriminating character of the all-pay auction. For example, when player 3 plays against the looser of the first match in the second match (node $D$ ), he does not spend any effort because he already knows that his expected payoff from playing against the winner of the first match in match 3 (node $A$ or $B$ ) will be zero if it is organized as an all-pay auction. By contrast, in a non-perfectly discriminating contest, his expected payoff in match 3 (node $A$ or $B$ ) is positive and so is his effort in match 2 (node $D$ ), i.e. the discouragement of the trailing player is less pronounced. ${ }^{9}$

\footnotetext{
${ }^{9}$ Konrad (2009, Chapter 8.6) makes an analog argument for elimination tournaments.
} 


\subsubsection{Endogenous sequences}

The last observation suggests that the discouragement of player 3 may be substantially reduced if he does not face the winner of the first match in match 3 . Thus, an endogenous sequence of matches in which player 3 always faces the winner of the first match in match 2 and the loser of the first match in match 3, referred to as winner first (WF) and depicted in Figure 4, may significantly increase the fairness of the contest. ${ }^{10}$ Considering the endogenous

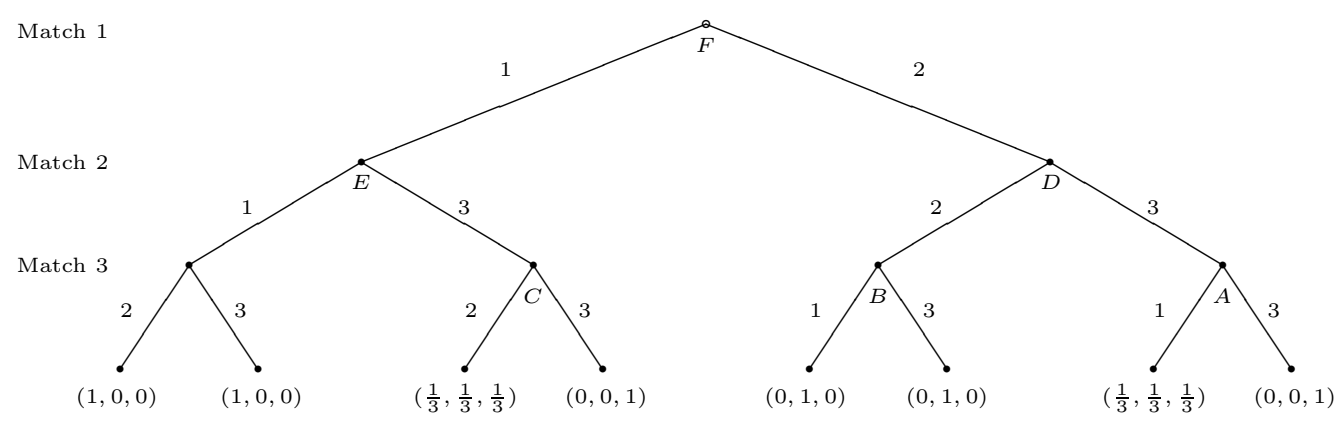

Figure 4 3-player round-robin tournament with endogenous sequence WF

WF-sequence and replicating the above calculations for the lottery tournament as well as those in Krumer et al. (2017) for the APA-tournament ${ }^{11}$ yields the equilibrium values of the ex-ante winning probabilities and expected payoffs displayed in the columns labelled endogenous WF of Tables 1 and $2 .{ }^{12}$ Indeed, the results demonstrate that endogenizing the sequence significantly decreases the level of discrimination in both cases: In the APAtournament, the relative standard deviation of ex-ante winning probabilities (expected payoffs) drops from $74.43 \%$ to $6.43 \%$ (108.01\% to $0 \%$ ), and in the lottery tournament from $6.12 \%$ to $2.30 \%$ (10.27\% to $3.00 \%)$.

Proposition 3 Suppose that there are 3 symmetric players and $r \in\{1, \infty\}$. Then the round-robin tournament with an endogenous WF-sequence is less discriminatory than the round-robin tournament with an exogenous sequence.

\footnotetext{
${ }^{10}$ In the 3 -player tournament, the only alternative endogenous sequence of matches is one in which player 3 always faces the winner of the first match in match 3 and the loser of the first match in match 2, referred to as loser first (LF). For the reasons just explained, the LF-sequence does not entail a similar increase in the fairness of the tournament. Details are provided by the author upon request. Notice, however that both endogenous sequences, WF and LF, treat players 1 and 2 in a perfectly symmetric way and thus leave them with the same ex-ante winning probabilities and expected payoffs.

${ }^{11}$ Baye et al. (1996) provide a full characterization of equilibria in all-pay auctions.

${ }^{12}$ Detailed calculations are provided by the author upon request.
} 


\subsubsection{Ex-ante effort provision}

The previous analysis has assumed that players are able to choose matchdependent effort levels, i.e. effort levels that may vary from match to match in the course of the tournament. If effort is interpreted as preparation time, e.g. days or weeks spent in a training camp in order to reach a certain fitness level, however, the schedule of many tournaments in sports does not allow players to adjust this level from match to match. Instead, players must decide beforehand how much time they invest in preparation and, once the tournament has started, compete in all of their matches with the same fitness level acquired.

I illustrate the impact of such ex-ante effort provision reconsidering the Tullock tournament with three players and an exogenous sequence of matches (Figure 1), but now assuming that the players must choose a single effort level for both of their matches before the tournament starts. Despite the fact that the order of their matches is asymmetric by the very nature of the tournament, the players' objective functions are then symmetric. It is straightforward to show that player $i$ 's expected payoff equals

$$
E_{i}=\frac{x_{i}^{2 r}}{\left(x_{i}^{r}+x_{j}^{r}\right)\left(x_{i}^{r}+x_{k}^{r}\right)}+\frac{2}{3} \frac{x_{i}^{r} x_{j}^{r} x_{k}^{r}}{\left(x_{i}^{r}+x_{j}^{r}\right)\left(x_{i}^{r}+x_{k}^{r}\right)\left(x_{j}^{r}+x_{k}^{r}\right)}
$$

for any $r>0$ and $i, j, k \in\{1,2,3\}$ with $i \neq j \neq k \neq i$. The necessary conditions $\frac{\partial E_{i}}{\partial x_{i}}=0$ imply $x_{i}=r / 4$ for all $i \in\{1,2,3\}$ in any symmetric pure-strategy Nash equilibrium. For $r \leq 4 / 3$, the players' resulting expected payoffs are non-negative and the sufficient condition for a Nash equilibrium in pure strategies is met.

Proposition 4 The sequential round-robin tournament with three symmetric players and ex-ante effort provision will be perfectly fair if its matches are organized as Tullock contests. For $r \leq 4 / 3$, a symmetric Nash equilibrium in pure strategies exists with $x_{i}=r / 4$ for all $i \in\{1,2,3\}$.

\subsection{Aggregate effort}

A major part of the literature on contests deals with the issue of rent dissipation, i.e. with the question how much of the rent is dissipated in form of aggregate effort in the course of the contest. Depending on the viewpoint of the contest designer, aggregate effort may be regarded as a waste of resources, e.g. in in political or military conflicts, or a valuable input that intensifies competition and attracts attention, like in sports or music contests. In this subsection, I compare aggregate effort provision for different forms of competition between three symmetric players. 


\subsubsection{Tullock tornaments vs. APA-tournaments}

In a static winner-take-all contest between three symmetric players, the rent is fully dissipated in an all-pay auction but only partially in a Tullock contest. If the rent is normalized to 1 , the expected aggregate effort in a mixedstrategy Nash equilibrium of the all-pay auction equals 1 . In the unique pure-strategy Nash equilibrium of the lottery contest, each player invests $2 / 9$, so aggregate effort equals $2 / 3$. Thus, the static competition of an allpay auction is more intense than that of a lottery contest.

By contrast, expected aggregate effort in sequential round-robin tournaments with all-pay auctions may be lower than in sequential round-robin tournaments with Tullock contests due to stronger discouragement effects in the former. For example, Table 2 shows that, for three players and an exogenous sequence of matches, the aggregate expected payoffs are larger and hence aggregate expected efforts are smaller in the APA-tournament than in the lottery tournament. Furthermore, Figure 3 implies that this result holds for the comparison of the APA-tournament with all Tullock tournaments with a discriminating power between a certain threshold $\hat{r}$ and $\bar{r} .{ }^{13}$

Proposition 5 Suppose that there are 3 symmetric players, an exogenous sequence of matches, and $r \leq \bar{r}$. Then expected aggregate effort is smaller in the APA-tournament than in the Tullock tournament if $r$ exceeds a certain threshold $\hat{r}<1$.

This implies that, for competition between three symmetric players, the contest designer can choose sequential round-robin tournaments with Tullock contests which are not only more balanced but also more intense than sequential round-robin tournaments with all-pay auctions.

\subsubsection{Endogenous sequences}

Table 2 also shows that, for three players and the endogenous WF-sequence of matches, the intensity of both the APA-tournament and the lottery tournament increases. In fact, in the APA-tournament the rent is fully dissipated (as it is in the static all-pay auction), i.e. for the the endogenous WF-sequence the APA-tournament is more intense than the lottery tournament.

Proposition 6 Suppose that there are 3 symmetric players and $r \in\{1, \infty\}$. Then the round-robin tournament with an endogenous WF-sequence is more intense than the round-robin tournament with an exogenous sequence. Moreover, with an endogenous WF-sequence, expected aggregate effort in the APAtournament equals the rent and is larger than in the lottery tournament.

\footnotetext{
${ }^{13}$ The simulations show that $\hat{r} \approx 0.72$.
} 
For competition between three symmetric players, this implies that the contest designer can improve both, the fairness and the intensity of competition, by using the endogenous WF-sequence instead of the exogenous sequence of matches. Notice, however, that for both, the exogenous and the endogenous WF-sequence, aggregate expected effort in the round-robin tournament with three symmetric players is never larger than in the respective static contest.

\section{The Tournament with Four Players}

In this section, I consider round-robin tournaments with four players. For the sake of concreteness, I focus on lottery tournaments, i.e. I assume $r=1$. $^{14}$ Again, I first determine the subgame perfect equilibrium of the tournament with a certain exogenously given sequence of matches organized as lottery contests, and then use the results to discuss the issues of fairness and effort provision, particularly in comparison to the respective APA-tournament.

\subsection{Lottery tournament: analysis and equilibrium}

In the tournament with four players, 30 different exogenous sequences of matches (except for renaming players) may be considered. Due to their empirical predominance, I follow Krumer et al. (2017) and focus primarily on 4-player tournaments that are organized in three consecutive rounds of two sequential matches with disjunct players. Under this restriction, there is always one player who has the first match in the first and second round and one player who has the second match in the first and second round. Hence, without further loss of generality, the analysis can be restricted to two possible sequences that differ only in the order of the matches in the third round.

In what follows, I present only the analysis for the sequence in which player 1 has the first match in all three rounds. More precisely, first player 1 is matched with player 2, second player 3 is matched with player 4 , third player 1 is matched with player 3 , fourth player 2 is matched with player 4 , fifth player 1 is matched with player 4 , and sixth player 2 is matched with player $3 .{ }^{15}$ The structure of the resulting sequential game is depicted

\footnotetext{
${ }^{14} \mathrm{As}$ in the case with three players, simulations show that similar results hold for all $r$ sufficiently small for a subgame perfect equilibrium in pure strategies to exist. Details are provided by the author upon request.

${ }^{15}$ Krumer et al. (2017) refer to this sequence as case A. Just as in their paper, the analysis of the sequence in which the order of the last two matches is reversed (case B) leads to the same outcome as in case A, confirming their invariance result. The calculations are provided upon request.
} 
in Figure 5 in Appendix C. ${ }^{16}$ The nodes $k \in\{1, \ldots, 55\}$ represent the nontrivial combinations for which the winner of the tournament has not yet been determined when the respective match starts.

Again I solve the game by backward induction for its subgame perfect equilibrium, making repeatedly use of equations (3), (4), and (1). The detailed derivation of the equilibrium values can be found in Appendix B. The columns labelled Case $A / B$ of Tables 3 and 4 summarize the equilibrium values of the players' ex-ante winning probabilities and expected payoffs for the APA-tournament (Krumer et al., 2017) and the the Tullock tournament with lottery contests (LC).

Table 3 Winning probabilities in 4-player tournaments

\begin{tabular}{l|cc|cc}
\hline \hline sequence & \multicolumn{2}{|c|}{ Case A/B } & \multicolumn{2}{c}{ Case C } \\
\hline matches & APA & LC & APA & LC \\
\hline player 1 & 0.621 & 0.2483 & 0.1423 & 0.2022 \\
player 2 & 0.051 & 0.2516 & 0.3839 & 0.2276 \\
player 3 & 0.252 & 0.2497 & 0.4051 & 0.2462 \\
player 4 & 0.076 & 0.2504 & 0.0686 & 0.3241 \\
\hline rel. stand. dev. & 0.9111 & 0.0048 & 0.5882 & 0.1821 \\
\hline \hline
\end{tabular}

Table 4 Expected payoffs in 4-player tournaments

\begin{tabular}{l|cc|cc}
\hline \hline sequence & \multicolumn{2}{|c|}{ Case A/B } & \multicolumn{2}{c}{ Case C } \\
\hline matches & APA & LC & APA & LC \\
\hline player 1 & 0.300 & 0.0658 & 0 & 0.0393 \\
player 2 & 0.039 & 0.0687 & 0.1111 & 0.0691 \\
player 3 & 0.009 & 0.0669 & 0.0849 & 0.0645 \\
player 4 & 0.001 & 0.0687 & 0 & 0.1038 \\
\hline rel. stand. dev. & 1.4171 & 0.0182 & 1.0178 & 0.3322 \\
$\sum$ expected payoffs & 0.349 & 0.2701 & 0.1960 & 0.2767 \\
\hline \hline
\end{tabular}

Proposition 7 In the unique subgame perfect equilibrium of the sequential round-robin tournament with four symmetric players and matches organized as lottery contests in one of the sequences $A$ or $B$, the player who competes

\footnotetext{
${ }^{16}$ See also Krumer et al. (2017, Figures 2 and 3).
} 
in the first matches of each of the first two rounds (player 1) has the lowest probability to win as well as the lowest expected payoff. Quantitatively, however, differences in winning probabilities and expected payoffs are very small, i.e. the tournament is almost fair.

\subsection{Discussion}

Again, in comparison to the findings by Krumer et al. (2017) for matches organized as all-pay auctions, the results for matches organized as lottery contests differ fundamentally in both, the quality and the quantity of discrimination induced by the sequential order of matches. Qualitatively, the player who competes in the first matches of each of the first two rounds (player 1) is the most favored player in all-pay tournaments, whereas he is the least favored player in lottery tournaments. Quantitatively, the relative standard deviations of the ex-ante expected winning probabilities and expected payoffs are much smaller for the lottery tournament than the APA-tournament. Also the results with respect to aggregate expected effort resemble the case with three players: Aggregate ex-ante expected payoffs are smaller and hence aggregate expected efforts are larger in the lottery tournament than in the APA-tournament.

Proposition 8 Suppose that there are 4 symmetric players and an exogenous sequence of matches as in cases $A$ or $B$. Then the lottery tournament is less discriminatory than the APA-tournament as measured by both, the relative standard deviation of ex-ante winning probabilities and the relative standard deviation of ex-ante expected payoffs. Moreover, the lottery tournament is more intense as measured by ex-ante expected aggregate efforts.

Krumer et al. (2017) refer to the result, that the player who competes in the first matches of each of the first two rounds (player 1) is the most favored player for APA-tournaments with exogenous sequences as in cases A and $\mathrm{B}$, as first-mover advantage. In what follows, I show that this notion is misleading. To this end, consider the alternative exogenous sequence of matches in which first player 1 is matched with player 2 , second player 1 is matched with player 3 , third player 1 is matched with player 4 , fourth player 2 is matched with player 3 , fifth player 2 is matched with player 4 , and sixth player 3 is matched with player 4 . I refer to this alternative exogenous sequence, in which player 1 has his three matches as early as possible, as case $\mathrm{C}$. The respective structure of the tournament is depicted in Figure 6 in Appendix C.

Replicating the above calculations for the lottery tournament as well as those in Krumer et al. (2017) for the APA-tournament yields the equilibrium 
values of the ex-ante winning probabilities and expected payoffs displayed in the columns labelled Case $C$ of Tables 3 and $4 .{ }^{17}$ It becomes clear that player 1 neither has the highest ex-ante expected winning probability nor the highest ex-ante expected payoff despite the fact that he makes the first three moves, i.e. takes part in the first three matches. In the lottery tournament of case $\mathrm{C}$, he is even the least favored player. The example illustrates that finding a general rule about the direction of discrimination in sequential round robin tournaments with (more than) four players is a non-trivial task. It seems, however, to be a general rule that the lottery tournament (or more generally any Tullock tournament with sufficiently low discriminating power $r$ ) is less discriminatory than the APA-tournament.

\section{Conclusion}

I examined sequential round-robin tournaments with three and four symmetric players in which each player is matched once with each other player. I showed that if the matches are organized as Tullock contests (all-pay auctions), the tournament will be almost fair (highly discriminatory): Subject to the position of their matches in the sequence of the tournament, the differences in players' ex-ante winning probabilities and expected payoffs will be small (large). The differing results originate from the higher discriminating power of the all-pay auction. Moreover, the resulting discouragement effect in tournaments with all-pay auctions implies lower aggregate effort than in tournaments with Tullock contests.

Because most contests in sports inherently contain some source of randomness, the highest effort does not always prevail. A less than fully discriminating contest success function may, hence, be a more suitable description of real competition in sports. Moreover, the schedule of many tournaments requires that participants fix (a major part of) their effort in advance. In this sense, discrimination by the order of matches in sequential round-robin tournaments seems to be a minor problem in practice.

This conclusion is challenged in a recent working paper by Krumer and Lechner (2016) who find some empirical evidence for discrimination in roundrobin sports tournaments. The data they use does, however, not perfectly fit the model presented here. Laboratory experiments may help to better control the data generating process and test some of the paper's theoretical predictions.

\footnotetext{
${ }^{17}$ Detailed calculations are provided by the author upon request.
} 


\section{Appendix}

\section{A Backward Induction of the 3-Player Lottery Tournament}

3rd stage: player 2 vs. player 3

In node $A$, player 2 has won the first match and player 3 has won the second match. Thus $w_{2}^{A}=w_{3}^{A}=1$ and $\ell_{2}^{A}=\ell_{3}^{A}=0$, which yields $x_{2}^{A}=x_{3}^{A}=1 / 4$, $p_{2}^{A}=p_{3}^{A}=1 / 2$, and $E_{2}^{A}=E_{3}^{A}=1 / 4$.

In node $B$, player 2 has won the first match and player 1 has won the second match. Thus $w_{2}^{B}=1, w_{3}^{B}=1 / 3, \ell_{2}^{B}=1 / 3$, and $\ell_{3}^{B}=0$, which yields $x_{2}^{B}=4 / 27, x_{3}^{B}=2 / 27, p_{2}^{B}=2 / 3, p_{3}^{B}=1 / 3, E_{2}^{B}=17 / 27$, and $E_{3}^{B}=1 / 27$.

In node $C$, player 1 has won the first match and player 3 has won the second match. Thus $w_{2}^{C}=1 / 3, w_{3}^{C}=1, \ell_{2}^{C}=0$, and $\ell_{3}^{C}=1 / 3$, which yields $x_{2}^{C}=2 / 27, x_{3}^{C}=4 / 27, p_{2}^{C}=1 / 3, p_{3}^{C}=2 / 3, E_{2}^{C}=1 / 27$, and $E_{3}^{C}=17 / 27$.

2nd stage: player 1 vs. player 3

In node $D$, player 2 has won the first match. Thus $w_{1}^{D}=\frac{1}{3} p_{3}^{B}=1 / 9$, $w_{3}^{D}=E_{3}^{A}=1 / 4, \ell_{1}^{D}=0$, and $\ell_{3}^{D}=E_{3}^{B}=1 / 27$, which yields $x_{1}^{D}=92 / 3675$, $x_{3}^{D}=529 / 11025, p_{1}^{D}=12 / 35, p_{3}^{D}=23 / 35, E_{1}^{D}=48 / 3675$, and $E_{3}^{D}=$ $5689 / 44100$.

In node $E$, player 1 has won the first match. Thus $w_{1}^{E}=1, w_{3}^{E}=$ $E_{3}^{C}=17 / 27, \ell_{1}^{E}=\frac{1}{3} p_{2}^{C}=1 / 9$, and $\ell_{3}^{E}=0$, which yields $x_{1}^{E}=1088 / 5043$, $x_{3}^{E}=2312 / 15129, p_{1}^{E}=24 / 41, p_{3}^{E}=17 / 41, E_{1}^{E}=6289 / 15129$, and $E_{3}^{E}=$ $4913 / 45387$.

1st stage: player 1 vs. player 2

In node $F, w_{1}^{F}=E_{1}^{E}=6289 / 15129, w_{2}^{E}=p_{1}^{D} E_{2}^{B}+p_{3}^{D} E_{2}^{A}=1437 / 3780$, $\ell_{1}^{F}=E_{1}^{D}=48 / 3675$, and $\ell_{2}^{F}=p_{3}^{E} E_{2}^{C}=17 / 1107$, which yields

$$
\begin{aligned}
& x_{1}^{F}=\frac{7248212107119019482368}{72247255168680294207735} \approx 0.1003, \\
& x_{2}^{F}=\frac{94540743268528827361}{1057276904907516500601} \approx 0.0894,
\end{aligned}
$$




$$
\begin{aligned}
p_{1}^{F} & =\frac{5730786048}{10838598233} \approx 0.5287, \\
p_{2}^{F} & =\frac{5107812185}{10838598233} \approx 0.4713, \\
E_{1}^{F} & =\frac{124036989445771408416208}{987379153971964020839045} \approx 0.1256, \\
E_{2}^{F} & =\frac{16967370418961634099817}{173393412404832706098564} \approx 0.0979 .
\end{aligned}
$$

Moreover, player 3's expected payoff equals

$$
E_{3}^{F}=p_{1}^{F} E_{3}^{E}+p_{2}^{F} E_{3}^{D}=\frac{387078163887677}{3279543053341140} \approx 0.1180
$$

and the players' ex-ante winning probabilities are given by

$$
\begin{aligned}
& P_{1}=p_{1}^{F}\left(p_{1}^{E}+\frac{1}{3} p_{3}^{E} p_{2}^{C}\right)+\frac{1}{3} p_{2}^{F} p_{1}^{D} p_{3}^{B}=\frac{469024798252}{1333147582659} \approx 0.3518, \\
& P_{2}=\frac{1}{3} p_{1}^{F} p_{3}^{E} p_{2}^{F}+p_{2}^{F}\left[p_{1}^{D}\left(p_{2}^{B}+\frac{1}{3} p_{3}^{B}\right)+p_{3}^{D} p_{2}^{A}\right]=\frac{812878549919}{2666295165318} \approx 0.3049, \\
& P_{3}=p_{1}^{F} p_{3}^{E}\left(\frac{1}{3} p_{2}^{C}+p_{3}^{C}\right)+p_{2}^{F}\left(\frac{1}{3} p_{1}^{D} p_{3}^{B}+p_{3}^{D} p_{3}^{A}\right)=\frac{915367018895}{2666295165318} \approx 0.3433 .
\end{aligned}
$$

\section{B Backward Induction of the 4-Player Lottery Tournament}

6th stage: player 2 vs. player 3

Table 5 summarizes the results for the possible matches between player 2 and 3 in nodes 1 to 24 .

5th stage: player 1 vs. player 4

Table 6 summarizes the results for the possible matches between player 1 and 4 in nodes 25 to 40 .

4th stage: player 2 vs. player 4

Table 7 summarizes the results for the possible matches between player 2 and 4 in nodes 41 to 48 . 
Table 5 6th stage: player 2 vs. player 3

\begin{tabular}{r|cccccccccc}
\hline \hline$k$ & $w_{2}^{k}$ & $\ell_{2}^{k}$ & $w_{3}^{k}$ & $\ell_{3}^{k}$ & $x_{2}^{k}$ & $x_{3}^{k}$ & $p_{2}^{k}$ & $p_{3}^{k}$ & $E_{2}^{k}$ & $E_{3}^{k}$ \\
\hline 1 & $\frac{1}{2}$ & 0 & $\frac{1}{2}$ & 0 & $\frac{1}{8}$ & $\frac{1}{8}$ & $\frac{1}{2}$ & $\frac{1}{2}$ & $\frac{1}{8}$ & $\frac{1}{8}$ \\
2 & 0 & 0 & $\frac{1}{3}$ & 0 & 0 & 0 & 0 & 1 & 0 & $\frac{1}{3}$ \\
3 & $\frac{1}{3}$ & 0 & 1 & $\frac{1}{3}$ & $\frac{2}{27}$ & $\frac{4}{27}$ & $\frac{1}{3}$ & $\frac{2}{3}$ & $\frac{1}{27}$ & $\frac{17}{27}$ \\
4 & $\frac{1}{2}$ & 0 & 1 & $\frac{1}{2}$ & $\frac{1}{8}$ & $\frac{1}{8}$ & $\frac{1}{2}$ & $\frac{1}{2}$ & $\frac{1}{8}$ & $\frac{5}{8}$ \\
5 & 0 & 0 & 1 & $\frac{1}{2}$ & 0 & 0 & 0 & 1 & 0 & 1 \\
6 & 0 & 0 & 1 & $\frac{1}{2}$ & 0 & 0 & 0 & 1 & 0 & 1 \\
7 & $\frac{1}{3}$ & 0 & 0 & 0 & 0 & 0 & 1 & 0 & $\frac{1}{3}$ & 0 \\
8 & $\frac{1}{2}$ & 0 & $\frac{1}{2}$ & 0 & $\frac{1}{8}$ & $\frac{1}{8}$ & $\frac{1}{2}$ & $\frac{1}{2}$ & $\frac{1}{8}$ & $\frac{1}{8}$ \\
9 & $\frac{1}{2}$ & 0 & $\frac{1}{2}$ & 0 & $\frac{1}{8}$ & $\frac{1}{8}$ & $\frac{1}{2}$ & $\frac{1}{2}$ & $\frac{1}{8}$ & $\frac{1}{8}$ \\
10 & 0 & 0 & $\frac{1}{3}$ & 0 & 0 & 0 & 0 & 1 & 0 & $\frac{1}{3}$ \\
11 & 1 & $\frac{1}{3}$ & $\frac{1}{3}$ & 0 & $\frac{4}{27}$ & $\frac{2}{27}$ & $\frac{2}{3}$ & $\frac{1}{3}$ & $\frac{17}{27}$ & $\frac{1}{27}$ \\
12 & 1 & $\frac{1}{2}$ & $\frac{1}{2}$ & 0 & $\frac{1}{8}$ & $\frac{1}{8}$ & $\frac{1}{2}$ & $\frac{1}{2}$ & $\frac{5}{8}$ & $\frac{1}{8}$ \\
13 & $\frac{1}{2}$ & 0 & $\frac{1}{2}$ & 0 & $\frac{1}{8}$ & $\frac{1}{8}$ & $\frac{1}{2}$ & $\frac{1}{2}$ & $\frac{1}{8}$ & $\frac{1}{8}$ \\
14 & $\frac{1}{2}$ & 0 & $\frac{1}{2}$ & 0 & $\frac{1}{8}$ & $\frac{1}{8}$ & $\frac{1}{2}$ & $\frac{1}{2}$ & $\frac{1}{8}$ & $\frac{1}{8}$ \\
15 & 1 & 0 & 1 & 0 & $\frac{1}{4}$ & $\frac{1}{4}$ & $\frac{1}{2}$ & $\frac{1}{2}$ & $\frac{1}{4}$ & $\frac{1}{4}$ \\
16 & 1 & 0 & 1 & 0 & $\frac{1}{4}$ & $\frac{1}{4}$ & $\frac{1}{2}$ & $\frac{1}{2}$ & $\frac{1}{4}$ & $\frac{1}{4}$ \\
17 & $\frac{1}{2}$ & 0 & 1 & $\frac{1}{2}$ & $\frac{1}{8}$ & $\frac{1}{8}$ & $\frac{1}{2}$ & $\frac{1}{2}$ & $\frac{1}{8}$ & $\frac{5}{8}$ \\
18 & $\frac{1}{3}$ & 0 & 1 & $\frac{1}{3}$ & $\frac{2}{27}$ & $\frac{4}{27}$ & $\frac{1}{3}$ & $\frac{2}{3}$ & $\frac{1}{27}$ & $\frac{17}{27}$ \\
19 & 1 & $\frac{1}{2}$ & 0 & 0 & 0 & 0 & 1 & 0 & 1 & 0 \\
20 & 1 & $\frac{1}{2}$ & 0 & 0 & 0 & 0 & 1 & 0 & 1 & 0 \\
21 & $\frac{1}{3}$ & 0 & 0 & 0 & 0 & 0 & 1 & 0 & $\frac{1}{3}$ & 0 \\
22 & 1 & $\frac{1}{2}$ & $\frac{1}{2}$ & 0 & $\frac{1}{8}$ & $\frac{1}{8}$ & $\frac{1}{2}$ & $\frac{1}{2}$ & $\frac{5}{8}$ & $\frac{1}{8}$ \\
24 & 1 & $\frac{1}{3}$ & $\frac{1}{3}$ & 0 & $\frac{4}{27}$ & $\frac{2}{27}$ & $\frac{2}{3}$ & $\frac{1}{3}$ & $\frac{17}{27}$ & $\frac{1}{27}$ \\
\hline \hline & $\frac{1}{2}$ & 0 & $\frac{1}{2}$ & 0 & $\frac{1}{8}$ & $\frac{1}{8}$ & $\frac{1}{2}$ & $\frac{1}{2}$ & $\frac{1}{8}$ & $\frac{1}{8}$ \\
\hline
\end{tabular}


Table 6 th stage: player 1 vs. player 4

\begin{tabular}{r|cccccccccc}
\hline \hline$k$ & $w_{1}^{k}$ & $\ell_{1}^{k}$ & $w_{4}^{k}$ & $\ell_{4}^{k}$ & $x_{1}^{k}$ & $x_{4}^{k}$ & $p_{1}^{k}$ & $p_{4}^{k}$ & $E_{1}^{k}$ & $E_{4}^{k}$ \\
\hline 25 & 1 & $\frac{1}{2}$ & 0 & 0 & 0 & 0 & 1 & 0 & 1 & 0 \\
26 & 1 & $\frac{1}{3}$ & $\frac{1}{3}$ & 0 & $\frac{4}{27}$ & $\frac{2}{27}$ & $\frac{2}{3}$ & $\frac{1}{3}$ & $\frac{17}{27}$ & $\frac{1}{27}$ \\
27 & $\frac{1}{9}$ & 0 & 0 & 0 & 0 & 0 & 1 & 0 & $\frac{1}{9}$ & 0 \\
28 & 0 & 0 & 0 & 0 & 0 & 0 & $\frac{1}{2}$ & $\frac{1}{2}$ & 0 & 0 \\
29 & 1 & $\frac{1}{3}$ & $\frac{1}{3}$ & 0 & $\frac{4}{27}$ & $\frac{2}{27}$ & $\frac{2}{3}$ & $\frac{1}{3}$ & $\frac{17}{27}$ & $\frac{1}{27}$ \\
30 & 1 & 0 & 1 & 0 & $\frac{1}{4}$ & $\frac{1}{4}$ & $\frac{1}{2}$ & $\frac{1}{2}$ & $\frac{1}{4}$ & $\frac{1}{4}$ \\
31 & $\frac{1}{2}$ & 0 & $\frac{1}{2}$ & 0 & $\frac{1}{8}$ & $\frac{1}{8}$ & $\frac{1}{2}$ & $\frac{1}{2}$ & $\frac{1}{8}$ & $\frac{1}{8}$ \\
32 & $\frac{1}{3}$ & 0 & 1 & $\frac{1}{3}$ & $\frac{2}{27}$ & $\frac{4}{27}$ & $\frac{1}{3}$ & $\frac{2}{3}$ & $\frac{1}{27}$ & $\frac{17}{27}$ \\
33 & $\frac{1}{9}$ & 0 & 0 & 0 & 0 & 0 & 1 & 0 & $\frac{1}{9}$ & 0 \\
34 & $\frac{1}{2}$ & 0 & $\frac{1}{2}$ & 0 & $\frac{1}{8}$ & $\frac{1}{8}$ & $\frac{1}{2}$ & $\frac{1}{2}$ & $\frac{1}{8}$ & $\frac{1}{8}$ \\
35 & 0 & 0 & 0 & 0 & 0 & 0 & $\frac{1}{2}$ & $\frac{1}{2}$ & 0 & 0 \\
36 & 0 & 0 & $\frac{1}{9}$ & 0 & 0 & 0 & 0 & 1 & 0 & $\frac{1}{9}$ \\
37 & 0 & 0 & 0 & 0 & 0 & 0 & $\frac{1}{2}$ & $\frac{1}{2}$ & 0 & 0 \\
38 & $\frac{1}{3}$ & 0 & 1 & $\frac{1}{3}$ & $\frac{2}{27}$ & $\frac{4}{27}$ & $\frac{1}{3}$ & $\frac{2}{3}$ & $\frac{1}{27}$ & $\frac{17}{27}$ \\
39 & 0 & 0 & $\frac{1}{9}$ & 0 & 0 & 0 & 0 & 1 & 0 & $\frac{1}{9}$ \\
40 & 0 & 0 & 1 & $\frac{1}{2}$ & 0 & 0 & 0 & 1 & 0 & 1 \\
\hline \hline
\end{tabular}

Table 7 4th stage: player 2 vs. player 4

\begin{tabular}{r|cccccccccc}
\hline \hline$k$ & $w_{2}^{k}$ & $\ell_{2}^{k}$ & $w_{4}^{k}$ & $\ell_{4}^{k}$ & $x_{2}^{k}$ & $x_{4}^{k}$ & $p_{2}^{k}$ & $p_{4}^{k}$ & $E_{2}^{k}$ & $E_{4}^{k}$ \\
\hline 41 & 0 & 0 & $\frac{1}{27}$ & 0 & 0 & 0 & 0 & 1 & 0 & $\frac{1}{27}$ \\
42 & $\frac{1}{27}$ & 0 & 0 & 0 & 0 & 0 & 1 & 0 & $\frac{1}{27}$ & 0 \\
43 & $\frac{1}{9}$ & 0 & $\frac{1}{4}$ & $\frac{1}{27}$ & $\frac{92}{3675}$ & $\frac{529}{11025}$ & $\frac{12}{35}$ & $\frac{23}{35}$ & $\frac{16}{1225}$ & $\frac{5689}{44100}$ \\
44 & $\frac{1}{8}$ & 0 & $\frac{17}{27}$ & $\frac{1}{8}$ & $\frac{2943}{147968}$ & $\frac{11881}{147968}$ & $\frac{27}{136}$ & $\frac{109}{136}$ & $\frac{729}{147968}$ & $\frac{179421}{3995136}$ \\
45 & $\frac{17}{27}$ & $\frac{1}{8}$ & $\frac{1}{8}$ & 0 & $\frac{11881}{147968}$ & $\frac{2943}{147968}$ & $\frac{109}{136}$ & $\frac{27}{136}$ & $\frac{1794421}{3995136}$ & $\frac{729}{147968}$ \\
46 & $\frac{1}{4}$ & $\frac{1}{27}$ & $\frac{1}{9}$ & 0 & $\frac{529}{11025}$ & $\frac{92}{3675}$ & $\frac{23}{35}$ & $\frac{12}{35}$ & $\frac{5689}{44100}$ & $\frac{16}{1225}$ \\
47 & 1 & $\frac{1}{9}$ & $\frac{17}{27}$ & 0 & $\frac{1088}{5043}$ & $\frac{2312}{15129}$ & $\frac{24}{41}$ & $\frac{17}{41}$ & $\frac{6289}{15129}$ & $\frac{4913}{45387}$ \\
48 & $\frac{17}{27}$ & 0 & 1 & $\frac{1}{9}$ & $\frac{2312}{15129}$ & $\frac{1088}{5043}$ & $\frac{17}{41}$ & $\frac{24}{41}$ & $\frac{4913}{45387}$ & $\frac{6289}{15129}$ \\
\hline \hline
\end{tabular}


3rd stage: player 1 vs. player 3

Table 8 summarizes the results for the possible matches between player 1 and 3 in nodes 49 to $52 .{ }^{18}$

Table 8 3rd stage: player 1 vs. player 3

\begin{tabular}{r|cccccccccc}
\hline \hline$k$ & $w_{1}^{k}$ & $\ell_{1}^{k}$ & $w_{3}^{k}$ & $\ell_{3}^{k}$ & $x_{1}^{k}$ & $x_{3}^{k}$ & $p_{1}^{k}$ & $p_{3}^{k}$ & $E_{1}^{k}$ & $E_{3}^{k}$ \\
\hline 49 & $\frac{17}{27}$ & $\frac{3}{27}$ & $\frac{17}{27}$ & $\frac{3}{27}$ & $\frac{7}{54}$ & $\frac{7}{54}$ & $\frac{1}{2}$ & $\frac{1}{2}$ & $\frac{13}{54}$ & $\frac{13}{54}$ \\
50 & $\frac{479}{1260}$ & $\frac{1601}{29376}$ & $\frac{1115}{9792}$ & 0 & $.063^{*}$ & $.022^{*}$ & $\frac{334829}{451904}$ & $\frac{117075}{451904}$ & $.233^{*}$ & $.008^{*}$ \\
51 & $\frac{1115}{9792}$ & 0 & $\frac{479}{1260}$ & $\frac{1601}{29376}$ & $.022^{*}$ & $.063^{*}$ & $\frac{117075}{451904}$ & $\frac{334829}{45104}$ & $.008^{*}$ & $.233^{*}$ \\
52 & $\frac{17}{1107}$ & 0 & $\frac{17}{1107}$ & 0 & $\frac{17}{4428}$ & $\frac{17}{4428}$ & $\frac{1}{2}$ & $\frac{1}{2}$ & $\frac{17}{4428}$ & $\frac{17}{4428}$ \\
\hline \hline
\end{tabular}

2nd stage: player 3 vs. player 4

In node $53, w_{3}^{53}=E_{3}^{49}=13 / 54, w_{4}^{53}=p_{1}^{50} E_{4}^{43}+p_{3}^{50} E_{4}^{44}=\frac{156247339377677}{737212325068800}$, $\ell_{3}^{53}=E_{3}^{50}=\frac{1698089946875}{222188341035008}$, and $\ell_{4}^{53}=p_{1}^{49} E_{4}^{41}+p_{3}^{49} E_{4}^{42}=1 / 54$, which yields

$$
\begin{aligned}
x_{3}^{53} & =\frac{2838592300722474040222650362146749734654634775}{49135791034944597473551108001886056745092481024} \approx 0.0578, \\
x_{4}^{53} & =\frac{708874538967681641088412112936414299353727}{14787360989839579327474310225748175978377216} \approx 0.0479, \\
p_{3}^{53} & =\frac{1532693462551164725}{2804523095144408637} \approx 0.5465, \\
p_{4}^{53} & =\frac{1271829632593243912}{2804523095144408637} \approx 0.4535, \\
E_{3}^{53} & =\frac{32268865619510630319895266092551562934974898125}{417654223797029078525184418016031482333286088704} \approx 0.0773, \\
E_{4}^{53} & =\frac{2244061357539697012443635836562446772662241291}{38493349076676154936831563806400720593713190400} \approx 0.0583 .
\end{aligned}
$$

In node $54, w_{3}^{54}=E_{3}^{51}=\frac{16327048390727783}{69989327426027520}, w_{4}^{54}=p_{1}^{52} E_{4}^{47}+p_{3}^{52} E_{4}^{48}=\frac{290}{1107}$,

\footnotetext{
${ }^{18}$ I have executed the calculations using a mathematical software. Rounded values are provided and asterisked whenever the exact fractions are too bulky.
} 


$$
\begin{aligned}
\ell_{3}^{54}= & E_{3}^{52}=\frac{17}{4428}, \text { and } \ell_{4}^{54}=p_{1}^{51} E_{4}^{45}+p_{3}^{51} E_{4}^{46}=\frac{897254541427}{81912480563200}, \text { which yields } \\
x_{3}^{54} & =\frac{88799387968769188918516829317180765517015031752265071}{1551235297242395322643863894614708104378795841368326144} \\
& \approx 0.0572, \\
x_{4}^{54} & =\frac{1023298311277248529565623201836747217152409852513549}{16339488065770167415612148018973079629397102492815360} \\
& \approx 0.0626, \\
p_{3}^{54} & =\frac{1175229979616683122055}{2460973603420861073823} \approx 0.4775, \\
p_{4}^{54} & =\frac{1285743623804177951768}{2460973603420861073823} \approx 0.5225, \\
E_{3}^{54} & =\frac{4921365392724088164672172732676283496654799279604516035263}{87626179470628426985506583678995631400149419487214007222272} \\
& \approx 0.0562, \\
E_{4}^{54} & =\frac{73349936401334844833083970343119121093540849957712940531}{922985001859225216973099017295751322105383525614154055680} \\
& \approx 0.0795 .
\end{aligned}
$$

1st stage: player 1 vs. player 2

In node 55 ,

$$
\begin{aligned}
w_{1}^{55} & =p_{3}^{53} E_{1}^{49}+p_{4}^{53} E_{1}^{50} \\
& =\frac{2474331316538169727790747193601}{10424515924439782643590519357440} \approx 0.2374, \\
w_{2}^{55} & =p_{3}^{54}\left(p_{1}^{51} E_{2}^{45}+p_{3}^{51} E_{2}^{46}\right)+p_{4}^{54}\left(p_{1}^{52} E_{2}^{47}+p_{3}^{52} E_{2}^{48}\right) \\
& =\frac{89291457061090419218773261188853699}{375048720789298856045377987182428160} \approx 0.2381, \\
\ell_{1}^{55} & =p_{3}^{54} E_{1}^{51}+p_{4}^{54} E_{1}^{52} \\
& =\frac{201371096487294092458129360746857911}{35606306117556157613934977714220171264} \approx 0.0057, \\
\ell_{2}^{55} & =p_{3}^{53}\left(p_{1}^{49} E_{2}^{41}+p_{3}^{49} E_{2}^{42}\right)+p_{4}^{53}\left(p_{1}^{50} E_{2}^{43}+p_{3}^{50} E_{2}^{44}\right) \\
& =\frac{1656715486509656204967423421}{109803621566379951015018393600} \approx 0.0151,
\end{aligned}
$$


which yields

$$
\begin{aligned}
x_{1}^{55} & \approx 0.0579 \\
x_{2}^{55} & \approx 0.0557 \\
p_{1}^{55} & =\frac{2730407019806871026124174198126176300459178307775500559624935}{5358176048571487367703638298565166695147743104042716613637951} \\
& \approx 0.5096, \\
p_{2}^{55} & =\frac{2627769028764616341579464100438990394688564796267216054013016}{5358176048571487367703638298565166695147743104042716613637951} \\
& \approx 0.4904, \\
E_{1}^{55} & \approx 0.06582, \\
E_{2}^{55} & \approx 0.06872 .
\end{aligned}
$$

Moreover, the expected payoffs of player 3 and 4 equal

$$
\begin{aligned}
& E_{3}^{55}=p_{1}^{55} E_{3}^{53}+p_{2}^{55} E_{3}^{54} \approx 0.06691, \\
& E_{4}^{55}=p_{1}^{55} E_{4}^{53}+p_{2}^{55} E_{4}^{54} \approx 0.06868,
\end{aligned}
$$

and the players' ex-ante winning probabilities are given by

$$
\begin{aligned}
& P_{1} \approx 0.2483, \\
& P_{2} \approx 0.2516, \\
& P_{3} \approx 0.2497, \\
& P_{4} \approx 0.2504 .
\end{aligned}
$$

\section{Graphical Representation of 4-Player Tournaments}


Match

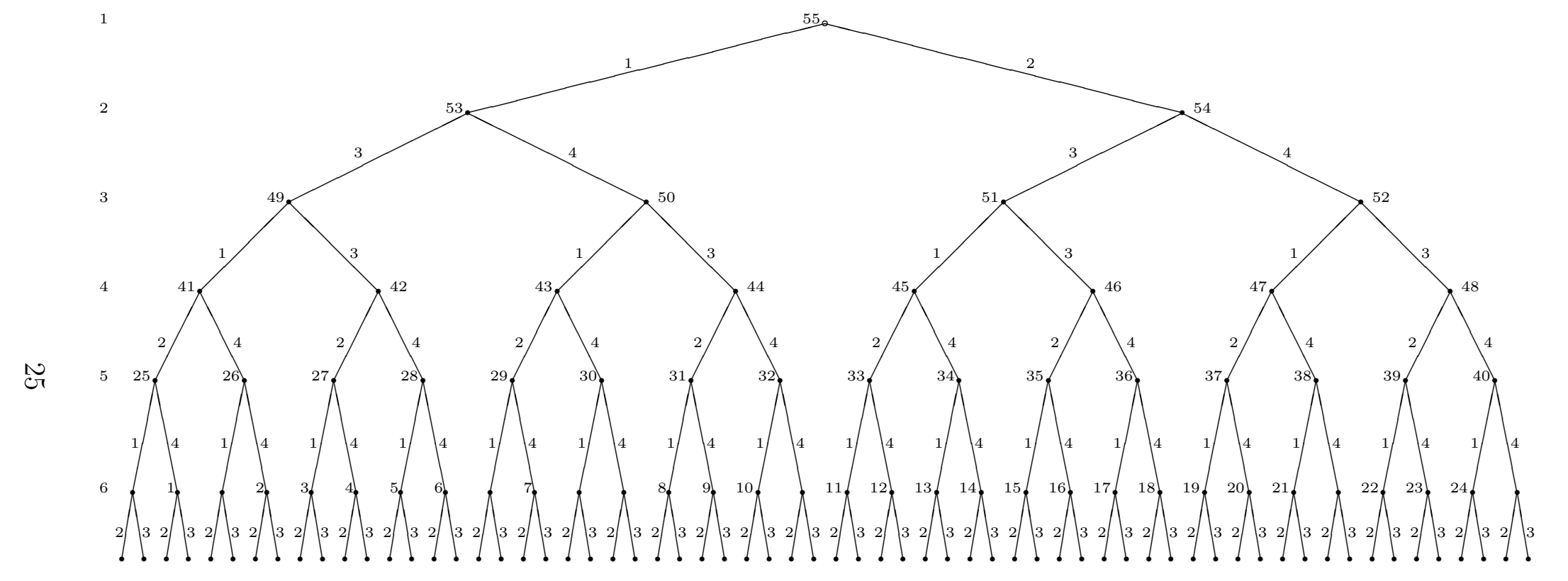

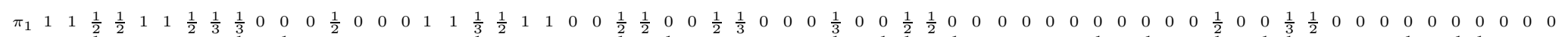

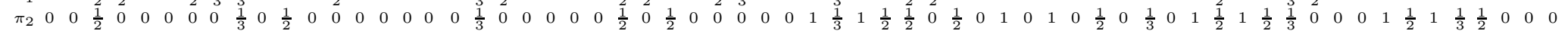

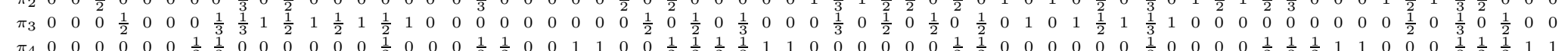

Figure 5 4-player round-robin tournament with exogenous sequence of case A: 1-2,3-4,1-3,2-4,1-4,2-3 
Match

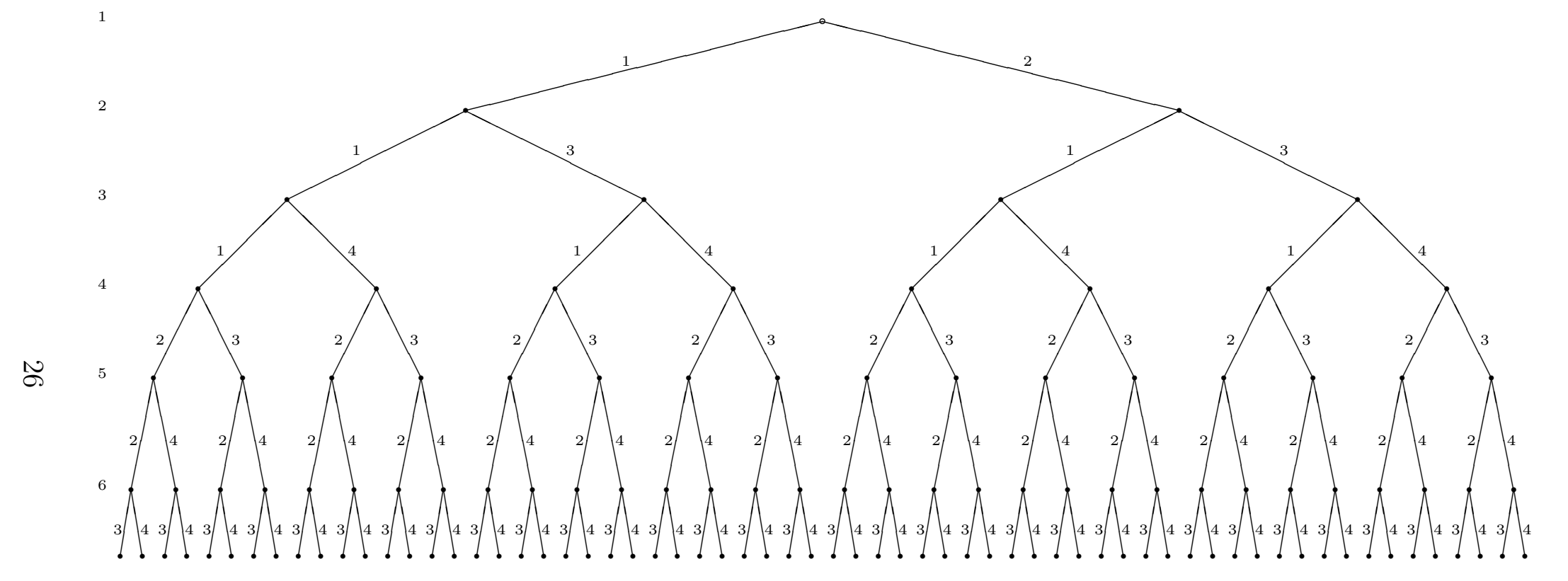

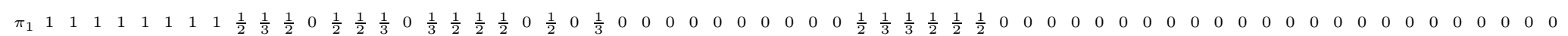

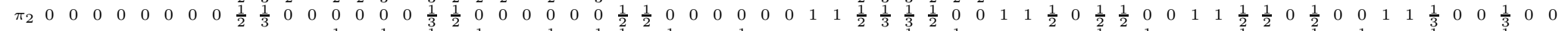

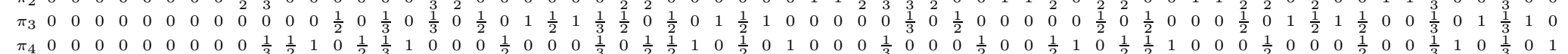

Figure 6 4-player round-robin tournament with exogenous sequence of case C: 1-2,1-3,1-4,2-3,2-4,3-4 


\section{References}

Baye, M. R., Kovenock, D., and de Vries, C. G. (1996). The all-pay auction with complete information. Economic Theory, 8(2):291-305.

Konrad, K. (2009). Strategy and Dynamics in Contests. Oxford University Press, Oxford.

Krumer, A. and Lechner, M. (2016). First in first win: Evidence on unfairness of round-robin tournaments in mega-events. Discussion Paper no. 2016-11, University of St. Gallen.

Krumer, A., Megidish, R., and Sela, A. (2017). First-mover advantage in round-robin tournaments. Social Choice and Welfare, 48(3):633-658.

Nti, K. O. (1999). Rent-seeking with asymmetric valuations. Public Choice, 98(3/4):415-430.

Skaperdas, S. (1996). Contest success functions. Economic Theory, 7(2):283290.

Szymanski, S. and Késenne, S. (2004). Competitive balance and gate revenue sharing in team sports. The Journal of Industrial Economics, 52(1):165177.

Tullock, G. (1980). Efficient rent seeking. In Buchanan, J., Tollison, R., and Tullock, G., editors, Towards a Theory of the Rent-Seeking Society, pages 97-112. Texas A\&M University Press, College Station, Tex. 